\title{
Improvement of Low-Frequency Radiated Emission in Electric Vehicle by Numerical Analysis
}

\author{
Feng Gao $\mathbb{D},{ }^{1}$ Chengkai Ye $\mathbb{D}^{1},{ }^{1}$ Zilong Wang, ${ }^{2}$ and ${\mathrm{Xu} \mathrm{Li}^{3}}^{3}$ \\ ${ }^{1}$ School of Automotive Engineering, Chongqing University, Chongqing 400044, China \\ ${ }^{2}$ China Automotive Technology and Research Center, Tianjin 300300, China \\ ${ }^{3}$ Chongqing Chang'an Automobile Corporation Limited, Chongqing 401120, China
}

Correspondence should be addressed to Feng Gao; gaofeng1@cqu.edu.cn

Received 22 March 2018; Revised 13 June 2018; Accepted 30 July 2018; Published 1 October 2018

Academic Editor: Daniela Proto

Copyright (C) 2018 Feng Gao et al. This is an open access article distributed under the Creative Commons Attribution License, which permits unrestricted use, distribution, and reproduction in any medium, provided the original work is properly cited.

\begin{abstract}
In this paper, a methodology for improving vehicle-level radiated electromagnetic interference (EMI) in electric vehicle $(\mathrm{EV})$ is proposed. This methodology predicts vehicle-level radiated EMI by using the multiport network theory, based on analyzing the contribution from each electronic component to find out the main EMI source. To validate its effectiveness, the proposed methodology is applied in an actual EV for low-frequency radiated emissions. Simulation in a commercially available electromagnetics software and measurement in the EV are combined to predict the vehicle-level emissions, and then the electronic component with the greatest EMI that causes failure to meet the EMC standard is identified. After improving this component, the vehicle-level radiated emission is reduced to comply with the EMC standard, proving that the presented numerical method is effective. The proposed methodology can also be used in other EMC issues, regardless of the amount of interference sources and sensitive equipment.
\end{abstract}

\section{Introduction}

In recent years, the problems of environmental pollution and energy crisis have aroused global concern. Compared to internal combustion engine vehicles, electric vehicles (EVs) have been rapidly developed because of such advantages as no pollution, no direct emission, and using renewable energy sources. However, high-voltage and current levels in EVs bring much more serious vehicle-level electromagnetic emission problems especially at low frequencies [1]. The electric traction system in EV is the source of high-voltage and high-current electromagnetic interference (EMI). It mainly consists of a high-voltage battery, a power distribution unit (PDU), a drive inverter, a direct-current/direct-current converter (DC/DC), an electric motor, and cables connecting the aforementioned components. The usage of power electronic devices in the drive inverter and the DC/DC causes large amounts of EMI because of high frequency switching [2]. Moreover, the cables connecting the components in the electric traction system carry the EMI and serve as the transmitting antenna to radiate the EMI to the environment [3].

To avoid disturbing other equipment, the EMI generated by EV is restricted to a certain level. EVs are obliged to pass such standards as CISPR 12 and J551-5 before they are put on the market. The former is for the high frequency band and the latter is for the low-frequency band [4]. The compliance of EV to these standards only can be verified at a very late stage in the vehicle development process, because the electromagnetic compatibility (EMC) is greatly affected by the design changes in electronic/electrical components, electrical systems, and metal structures. The EMC problem discovered lately requires more time and cost to resolve. Moreover, when an EMC problem is found, it is difficult and time consuming to identify the actual source of EMI because (1) there are many sources causing radiated EMI in EV; (2) the parasitic parameters among structures cause many hidden transmission paths or antennas radiating/transmitting EMI. Compared to the experimental tests, the prediction technique using numerical simulation is an effective way to analyze and 
improve the EMC of EV. Benefitting from the development of computer technologies and numerical solution algorithms for three-dimensional electromagnetic field with the coupling of cables, some commercial software packages for analysis of EMC have been presented, such as FEKO, CST Studio, and EMC Studio.

To improve low-frequency radiated emission in EV, automotive manufacturers usually troubleshoot the component with the greatest EMI and then improve the component-level EMC. Therefore, the present efforts in the literature on the improvement of radiated emission focus on component-level EMC. Di Piazza et al. developed a novel common-mode EMI active filter for automotive motor drives to mitigate the leakage high frequency common-mode currents, so as to reduce the radiated EMI [5]. Marczok et al. reworked the interference mechanisms of spark plugs and then improved its EMI properties so as to improve vehicle-level EMC [6]. Chun et al. proposed several solutions of switching noise reduction of DC/DC converter in HEV and EV for improvement of vehicle-level EMC [7]. These aforementioned works improve radiated emission through reduction of component-level emissions without consideration of the relationship between vehicle-level EMC and component-level EMC, so the degree of improvement is difficult to control. Numerical methods are efficient tools to analyze vehicle-level EMC issues. For analysis of vehicle-level radiated EMC issues using numerical methods, one widely used method is to establish the transfer function between EMI and the conducted interference current, which is measured in component test platforms or actual vehicles. Ala et al. predicted the radiated EMI generated by a $42 \mathrm{~V} / 14 \mathrm{~V}$ DC/DC step-down converter [8]. The transfer function from the time-domain interference current to the near electromagnetic field strength was calculated by finitedifference time-domain method. Chen et al. also anticipated the vehicle-level radiated EMI by characterizing the transfer function between the interference current carried in wire harnesses and the voltage at receiving antenna terminal [9]. Zeng et al. established the vehicle-level simulation model by using a three-dimensional electromagnetic analysis software package to calculate the conversion factor from the conductor current at the outputs of inverter to the voltage of antenna terminal [10]. Dong et al. obtained the coefficient between the electric field strength and the conducted current by analyzing the generation mechanism of radiated emissions [11]. Summing up the aforementioned studies, the transfer function should be obtained under the condition which is the same as the actual one, because the transfer function is greatly influenced by the impedance of source and victim. This poses great restrictions on its application in practice.

To improve the low-frequency radiated emission in an EV with less number of experiments and cost, a new numerical analysis method is presented to find out the main source, which has the following advantages: (a) the vehicle-level model of EMI is described by algebraic equations in frequency domain, by which the cost of computation is reduced; (b) the interactions of impedance among sources, paths, and victims are decoupled to integrate more approaches to set up the vehicle-level model of EMI; (c) it can be applied at the whole vehicle development stage by integrating different modeling approaches, test data, etc. This paper is organized as follows. Section 2 describes the studied EMC problem. Section 3 introduces the proposed numerical method used to model and analyze the problem. The application effect of this methodology on an EV is shown in Section 4. Finally, Section 5 concludes the paper.

\section{Problem Description}

SAE J551-5 limits the level of magnetic and electric emissions level from EVs over the frequency range from $150 \mathrm{kHz}$ to 30 $\mathrm{MHz}$. Figure 1 shows the test results of a four-seat sedan EV according to SAE J551-5. From Figure 1(b), it is found that the margin is sufficient at the low-frequency range, whereas it is narrow at the relative high frequency range and the magnetic field exceeds the limit about $10 \mathrm{~dB} \mu \mathrm{A} / \mathrm{m}$ at $13.8 \mathrm{MHz}$.

Vehicle-level EMC is affected by many factors, among which the performance of components is very important. Furthermore, at this stage, compared with redesigning the electrical systems or arranging the components, it costs less to improve the component-level EMC. Automotive manufacturers usually attempt to find out the component with the greatest EMI by experimental tests. The widely used way of troubleshooting is to do standard tests after turning off different suspected electronic/electrical components, until the vehicle-level EMC gets better. Troubleshooting with this method costs more than the standard EMC test due to the high cost of long-hours EMC test facility and the need for hours of testing. Additionally, this troubleshooting method cannot quantify the contribution of the components which cannot be turned off during the experimental test, such as the motor and motor controller of an EV.

To improve the vehicle-level radiated emission more efficiently, a numerical method will be introduced to model and find out the main emission sources in the EV. This methodology combines numerical simulation using electromagnetic software and experimental modeling approaches to predict vehicle-level radiated EMI and analyze the contribution from each electronic/electrical component. In other words, the greatest source of radiated EMI can be found by this numerical method without multiple experimental tests.

\section{Radiated EMI Modeling}

The low-frequency radiated emission is mainly caused by high-voltage cables which carry common-mode conducted interference current. To improve the vehicle-level radiated EMI by numerical method, a mathematical model to describe the EMC problem should be obtained first. Considering the fact that the electrically small component can be equivalent to a circuit, which is more effective to be solved numerically, a new strategy to model the EMC problem is presented as shown in Figure 2. The electronic/electrical components, transmission paths, and the measuring instruments constitute a multiport network system, in which each electrically small component or measuring instrument acts as a terminal. The electrically large parts including high-voltage cables, vehicle body, and other large structures are considered as a network which serves as a black box to describe the 


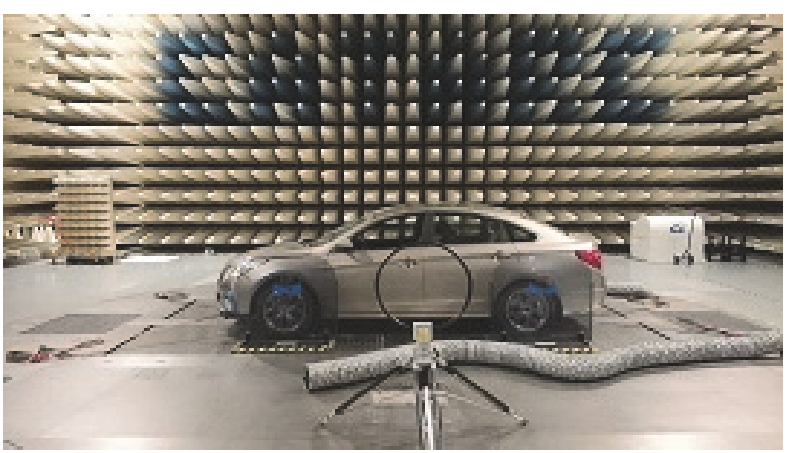

(a)

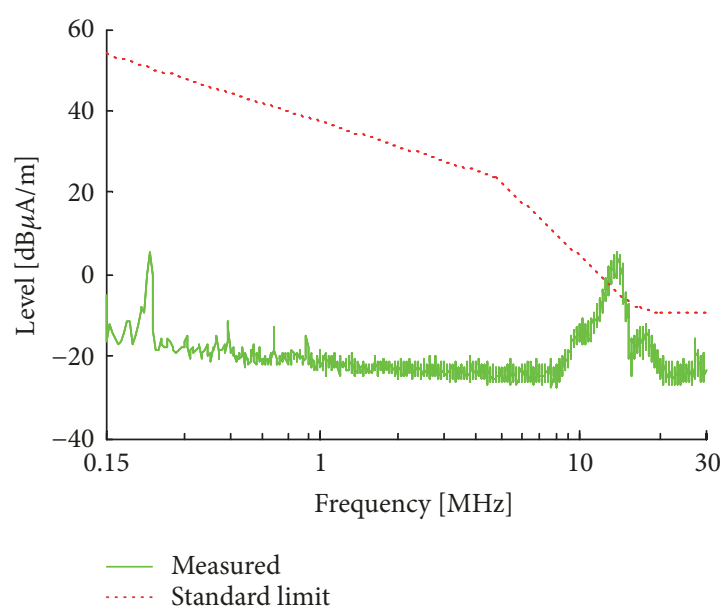

(b)

Figure 1: Experimental test of J551-5: (a) test setup; (b) test results.

transmission paths. The characteristics of the network can be calculated by existing commercial software or measured by network analyzer. Each terminal is characterized by Thevenin's equivalent circuit, whose circuit parameters can be measured in the EV.

The vehicle-level radiated emission is evaluated by the electromagnetic field strength measured by an antenna. Port 1 in Figure 2 denotes the measuring antenna while others (Indexed by $i=2, \cdots$ ) denote the on-board electronic components. Their Thevenin's equivalent circuits are described by the equivalent voltage source $V_{S, i}$ and Thevenin impedance $Z_{S, i}$. The port voltage and current are denoted by $U_{i}$ and $I_{i}$, respectively. Note that the equivalent voltage sources of the devices without electromagnetic interference, including the measuring antenna, are equal to zero.

The open-circuit impedance parameter called $\mathbf{Z}$ parameter is used to characterize the electromagnetic coupling relationship among the ports:

$$
\left[\begin{array}{c}
U_{1} \\
\hline U_{2} \\
\vdots \\
U_{n}
\end{array}\right]=\left[\begin{array}{c|ccc}
Z_{11} & Z_{12} & \cdots & Z_{1 n} \\
\hline Z_{21} & Z_{22} & \cdots & Z_{2 n} \\
\vdots & \vdots & \ddots & \vdots \\
Z_{n 1} & Z_{n 2} & \cdots & Z_{n n}
\end{array}\right]\left[\begin{array}{c}
I_{1} \\
I_{2} \\
\vdots \\
I_{n}
\end{array}\right]
$$

The vehicle-level emission is measured by the terminal voltage of the measuring antenna, and to distinguish the port of antenna from other on-board electronic/electrical components, (1) is rewritten using subscripts ant and ele:

$$
\left[\begin{array}{c}
U_{\text {ant }} \\
\mathbf{U}_{\text {ele }}
\end{array}\right]=\left[\begin{array}{ll}
Z_{\text {ant-ant }} & \mathbf{Z}_{\text {ant-ele }} \\
\mathbf{Z}_{\text {ele-ant }} & \mathbf{Z}_{\text {ele-ele }}
\end{array}\right]\left[\begin{array}{c}
I_{\text {ant }} \\
\mathbf{I}_{\text {ele }}
\end{array}\right]
$$

Combining all Thevenin's equivalent circuits of the terminals together yields

$$
\left[\begin{array}{c}
U_{\text {ant }} \\
\mathbf{U}_{\text {ele }}
\end{array}\right]=\left[\begin{array}{l}
V_{S, \text { ant }} \\
\mathbf{V}_{\text {S,ele }}
\end{array}\right]-\left[\begin{array}{ll}
Z_{S, \text { ant }} & \\
& \mathbf{Z}_{S, \text { ele }}
\end{array}\right]\left[\begin{array}{c}
I_{\text {ant }} \\
\mathbf{I}_{\text {ele }}
\end{array}\right]
$$

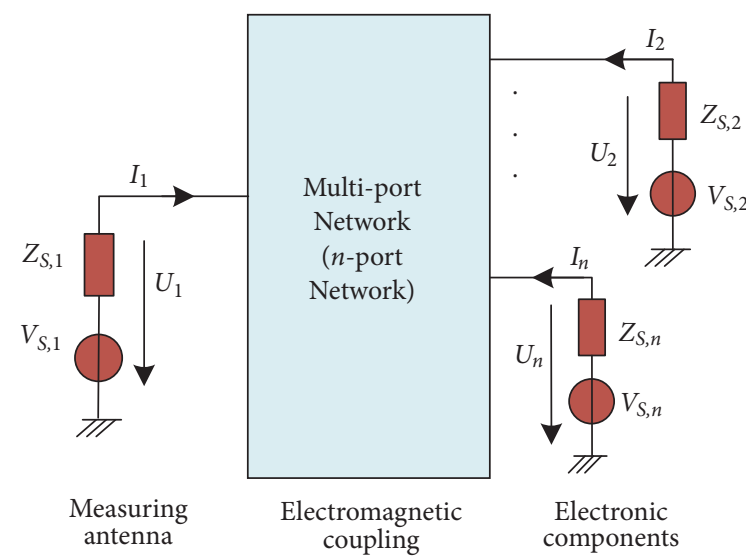

FIgURE 2: Model for pre-vehicle-level radiated EMI.

where $V_{S \text {,ant }}=0$ and $Z_{S \text {, ant }}=Z_{S, 1}$ are the equivalent voltage source and output impedances of measuring antenna. And $\mathbf{V}_{S, \text { ele }}=\left[V_{S, 2}, \cdots, V_{S, n}\right]^{\mathrm{T}}$ and $\mathbf{Z}_{S, \text { ele }}=\operatorname{diag}\left(Z_{S, 2}, \cdots, Z_{S, n}\right)$ denote the equivalent voltage sources and output impedances of the on-board electronic components connected to the ports. Equations (2) and (3), respectively, describe the electromagnetic coupling relationship between the multiple ports and the equivalent characteristics of on-board electronic components.

Based on (2) and (3), the voltages at each port can be derived by

$$
\begin{aligned}
& {\left[\begin{array}{c}
U_{\text {ant }} \\
\mathbf{U}_{\text {ele }}
\end{array}\right]} \\
& =\left[\begin{array}{cc}
Z_{\text {ant-ant }} & \mathbf{Z}_{\text {ant-ele }} \\
\mathbf{Z}_{\text {ele-ant }} & \mathbf{Z}_{\text {ele-ele }}
\end{array}\right] \\
& \cdot\left[\begin{array}{cc}
Z_{\text {ant-ant }}+Z_{S, a n t} & \mathbf{Z}_{\text {ant-ele }} \\
\mathbf{Z}_{\text {ele-ant }} & \mathbf{Z}_{\text {ele-ele }}+\mathbf{Z}_{\text {S,ele }}
\end{array}\right]^{-1}\left[\begin{array}{c}
V_{S, a n t} \\
\mathbf{V}_{\text {S,ele }}
\end{array}\right]
\end{aligned}
$$




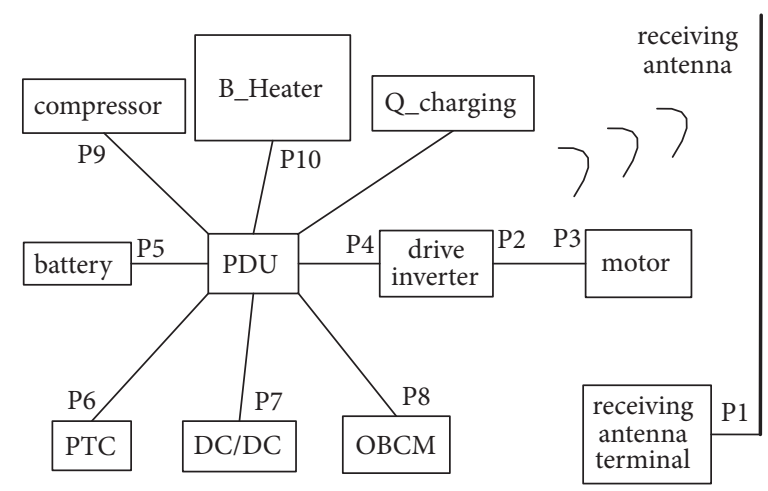

Figure 3: Multiport network of the EV.

Substituting the condition that $V_{S \text {,ant }}=0$ to (4), the terminal voltage of measuring antenna is

$$
\begin{gathered}
U_{\text {ant }}=\frac{Z_{S, a n t} \mathbf{Z}_{\text {ant-ele }}}{Z_{\text {ant-ant }}+Z_{S, a n t}}\left(\mathbf{Z}_{S, \text { ele }}+\mathbf{Z}_{\text {ele-ele }}\right. \\
\left.-\frac{\mathbf{Z}_{\text {ele-ant }} \mathbf{Z}_{\text {ant-ele }}}{Z_{\text {ant-ant }}+Z_{S, a n t}}\right)^{-1} \cdot \mathbf{V}_{S, \text { ele }}
\end{gathered}
$$

The conversion characteristics of measuring antenna are known in advance. The electromagnetic field strength at the specified position can be calculated by

$$
\begin{aligned}
|E| & =\left|U_{\text {ant }}\right| \cdot A F_{E} \\
\text { or }|H| & =\left|U_{\text {ant }}\right| \cdot A F_{H}
\end{aligned}
$$

where $|H|$ and $|E|$ denote the electric and magnetic field strength, respectively. $A F_{E}$ and $A F_{H}$ are the electric antenna factor and magnetic antenna factor, respectively. From (5) and (6), the electromagnetic field strength, which measures the vehicle-level radiated EMI, can be figured out by three parts: $\mathbf{Z}$-parameter of the entire network, the characteristics of electronic components, and measuring antenna. The above three parts can be obtained independently.

\section{Prediction Model Validation}

In this section, the predictive model is validated by applying it to predict the vehicle-level radiated EMI of the studied sedan EV. Figure 3 shows its equivalent multiport network for EMI prediction, which is a 10 -port network. The main components relating to EMI emission are determined from the previous standard and troubleshooting test results. Note that it is concluded from the previous results that the lowfrequency radiated EMI originates from the power electronic devices in the drive inverter and the DC/DC, so, in Figure 3, $\mathrm{P} 2, \mathrm{P} 4$, and $\mathrm{P} 7$ act as interference source.

4.1. Modeling of Port Coupling. As described in Section 3, Zparameter is used to characterize the port coupling of the 10 -port network. However, at the high frequency range, $\mathbf{S}$ parameter representation is more precise and easily obtained.
In this paper, the $\mathbf{S}$-parameter of the network is calculated by using FEKO, which is a commercial software package for numerical solving of three-dimensional electromagnetic field.

CAE preprocessing is necessary for EM simulation. It is widely accepted that the larger the EM energy is, the more detailed the EM model needs to be. Because the EMI source comes from drive inverter and the DC/DC and they are located at engine cabin, the EM energy mostly concentrates on engine cabin. In addition, the minor EM energy comes into the vehicle body because of the shielding effect of segregation board. So the EM model of engine cabin is detailed whereas the vehicle body can be highly simplified. The result of CAE preprocessing is shown in Figure 4(a). Figure 4(b) shows the calculation model in FEKO, which includes the main parts of EV and the measuring antenna arranged in the position required by SAE J551-5.

After calculating the $\mathbf{S}$-parameter of the network, the required Z-parameter can be calculated by [9].

$$
\begin{aligned}
& {\left[\begin{array}{cc}
Z_{\text {ant-ant }} & \mathbf{Z}_{\text {ant-ele }} \\
\mathbf{Z}_{\text {ele-ant }} & \mathbf{Z}_{\text {ele-ele }}
\end{array}\right]} \\
& =Z_{0}\left(\left[\begin{array}{ll}
1 & \\
& \mathbf{E}_{\text {ele }}
\end{array}\right]-\left[\begin{array}{ll}
S_{\text {ant-ant }} & \mathbf{S}_{\text {ant-ele }} \\
\mathbf{S}_{\text {ele-ant }} & \mathbf{S}_{\text {ele-ele }}
\end{array}\right]\right)^{-1} \\
& \cdot\left(\left[\begin{array}{ll}
1 & \\
& \mathbf{E}_{\text {ele }}
\end{array}\right]+\left[\begin{array}{ll}
S_{\text {ant-ant }} & \mathbf{S}_{\text {ant-ele }} \\
\mathbf{S}_{\text {ele-ant }} & \mathbf{S}_{\text {ele-ele }}
\end{array}\right]\right)
\end{aligned}
$$

where $Z_{0}$ denotes port reference impedance when calculating the $\mathbf{S}$-parameter, which is usually set to $50 \Omega$. And $\mathbf{E}_{\text {ele }}$ denotes the $9 \times 9$ identity matrix. Furthermore each part of $\boldsymbol{Z}$ parameter can be obtained:

$$
\begin{aligned}
& Z_{\text {ant-ant }}=Z_{0} \frac{1+S_{\text {ant-ant }}}{1-S_{\text {ant-ant }}}+2 Z_{0} \\
& -\mathbf{S}_{\text {ant-ele }}\left[\left(1-S_{\text {ant-ant }}\right)\left(\mathbf{E}_{\text {ele }}-\mathbf{S}_{\text {ele-ele }}\right)\right. \\
& \left.\quad-\mathbf{S}_{\text {ele-ant }} \mathbf{S}_{\text {ant-ele }}\right]^{-1} \cdot \mathbf{S}_{\text {ele-ant }} \\
& \mathbf{Z}_{\text {ant-ele }}=2 Z_{0} \cdot \mathbf{S}_{\text {ant-ele }}\left[\left(1-S_{\text {ant-ant }}\right)\left(\mathbf{E}_{\text {ele }}-\mathbf{S}_{\text {ele-ele }}\right)\right. \\
& \left.\quad-\mathbf{S}_{\text {ele-ant }} \mathbf{S}_{\text {ant-ele }}\right]^{-1}
\end{aligned}
$$




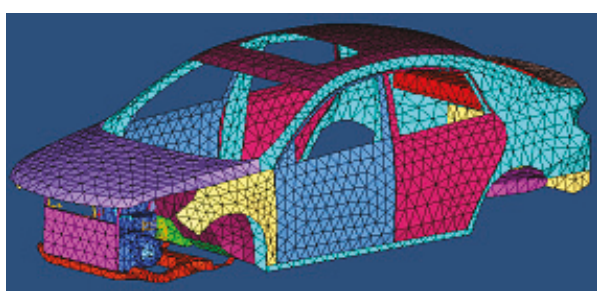

(a)

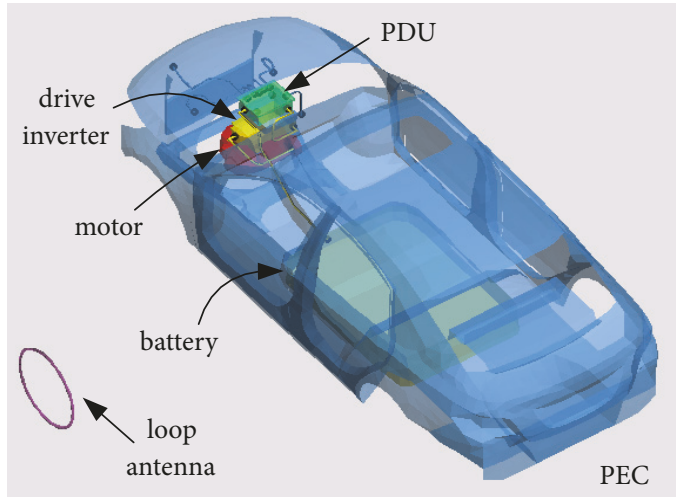

(b)

FIGURE 4: Simulation model: (a) CAE preprocessing result; (b) EM model in FEKO.

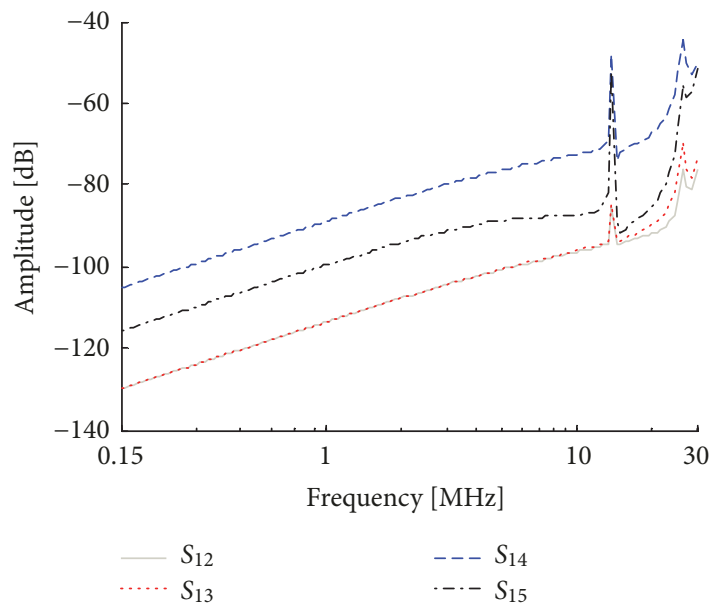

(a)

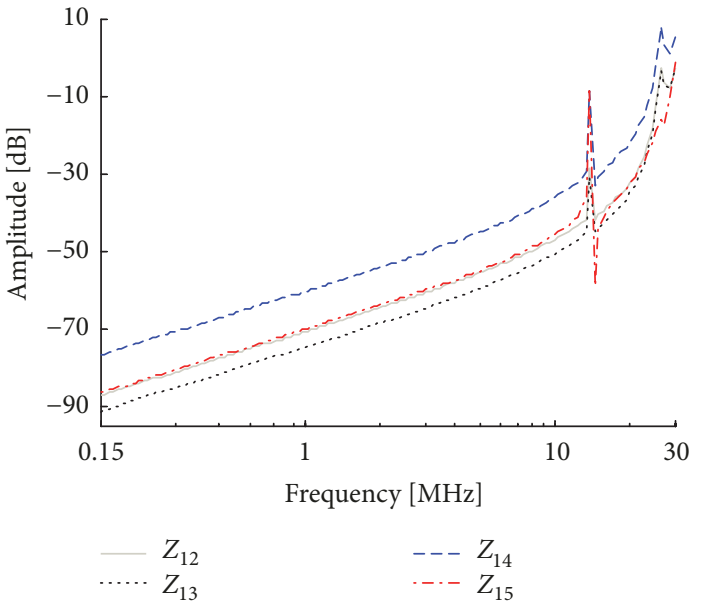

(b)

FIGURE 5: Network parameters: (a) S-parameter; (b) Z-parameter.

$$
\begin{aligned}
& \mathbf{Z}_{\text {ele-ant }}=2 Z_{0}\left[\left(1-S_{\text {ant-ant }}\right)\left(\mathbf{E}_{\text {ele }}-\mathbf{S}_{\text {ele-ele }}\right)\right. \\
& \left.\quad-\mathbf{S}_{\text {ele-ant }} \mathbf{S}_{\text {ant-ele }}\right]^{-1} \cdot \mathbf{S}_{\text {ele-ant }} \\
& \mathbf{Z}_{\text {ele-ele }}=Z_{0}\left[\left(1-S_{\text {ant-ant }}\right)\left(\mathbf{E}_{\text {ele }}-\mathbf{S}_{\text {ele-ele }}\right)\right. \\
& \left.\quad-\mathbf{S}_{\text {ele-ant }} \mathbf{S}_{\text {ant-ele }}\right]^{-1} \cdot\left[\left(1-S_{\text {ant-ant }}\right)\left(\mathbf{E}_{\text {ele }}+\mathbf{S}_{\text {ele-ele }}\right)\right. \\
& \left.\quad+\mathbf{S}_{\text {ele-ant }} \mathbf{S}_{\text {ant-ele }}\right]
\end{aligned}
$$

Equation (8) is essentially the conversion formula from $\mathbf{S}$-parameter to $\mathbf{Z}$-parameter for vehicle-level radiated EMI prediction. Figure 5 shows the $\mathbf{S}$-parameter and $\mathbf{Z}$-parameter curves which describe the relationship between the measuring antenna (Port 1) and some of the component ports (Port 2 to 5) as an example.

4.2. Equivalent Model of Components. The parameters of Thevenin's equivalent circuits for electronic/electrical components are measured in the actual EV, which is explained in Figure 6. Note that it is concluded from previous results

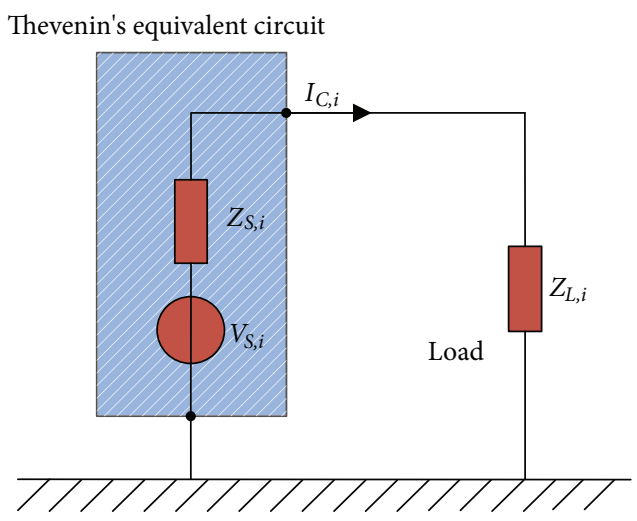

FIGURE 6: Measuring of electronic components.

that the radiated EMI is mainly caused by common-mode interferences. And so the positive and negative lines are equivalent to one. 


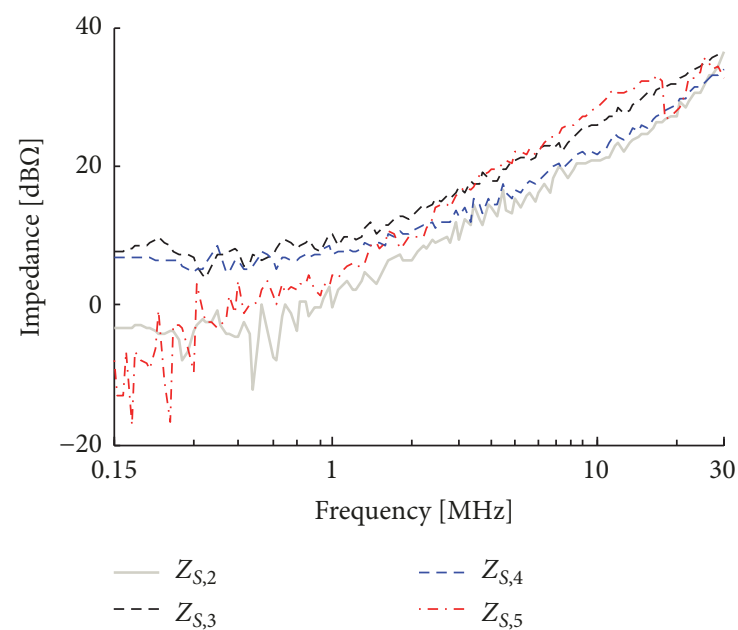

(a)

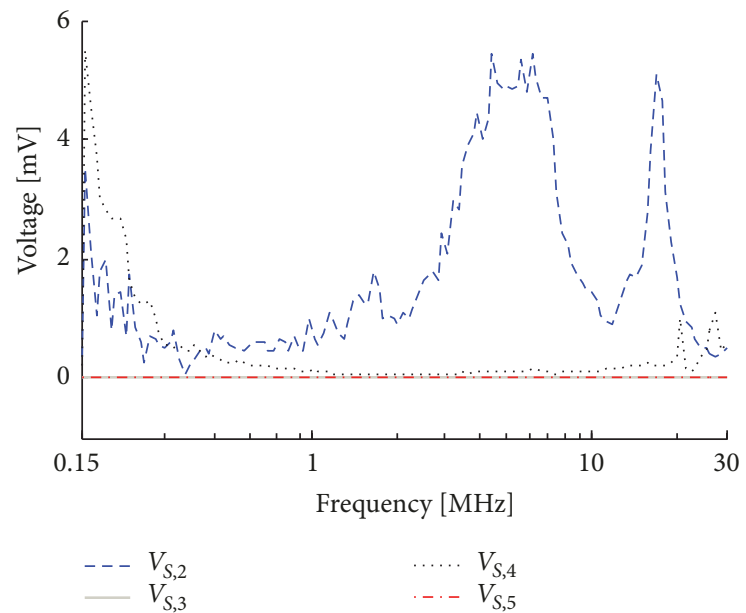

(b)

FIGURE 7: Thevenin's equivalent circuit parameters: (a) Thevenin impedance; (b) equivalent interference voltage.

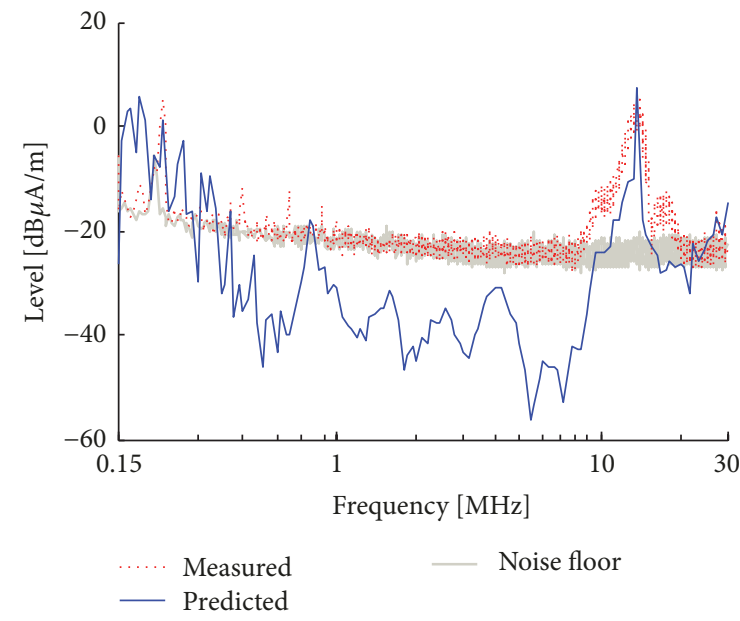

(a)

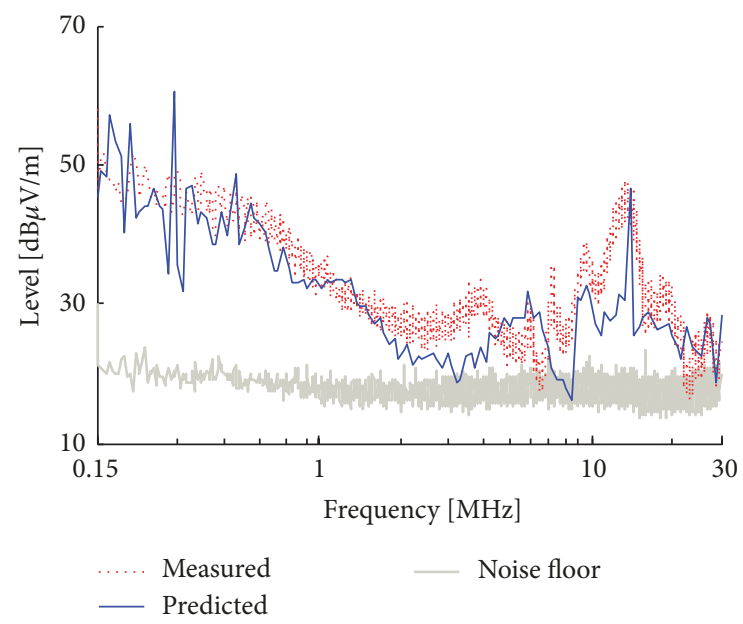

(b)

FIGURE 8: Predicted and measured EMI: (a) magnetic field; (b) electric field.

According to Figure 6, the parameters of Thevenin's equivalent circuit and the external load are formulated by

$$
V_{S, i}=I_{C, i} \bullet\left(Z_{S, i}+Z_{L, i}\right)
$$

where $I_{C, i}$ is the common-mode interference current, $V_{S, i}$ and $Z_{S, i}$ are the parameters of Thevenin's equivalent circuit, and $Z_{L, i}$ is the load impedance relative to ground. The interference current and impedance can be measured by a current clamp and a network analyzer, respectively. For the demonstration purpose, Figure 7 shows the output impedances and equivalent interference voltages of ports 2 to 5 .

4.3. Prediction Results and Analysis. With the derived parameters of the coupling characteristic between ports and Thevenin's equivalent circuit for components, the voltage at the measuring antenna terminal is calculated by (5). Furthermore, after calibrating the antenna property in FEKO, the radiated EMI is obtained using (6), which is compared with the measurement results as shown in Figure 8. The trend of the predicted and measured results agrees with each other. Moreover, the peak point of magnetic field at around 18.83 $\mathrm{MHz}$, which exceeds the limit, is predicted precisely. In the frequency range $0.3 \mathrm{kHz}-8 \mathrm{MHz}$ of the magnetic field, the predicted EMI is much smaller than the measured value on the whole. Comparing the measured value with the noise floor of EMC laboratory, we find that in the inaccurate frequency range the antenna only measures the noise floor, which implies that the emission generated by EV is much smaller.

\section{Main EMI Analysis and Improvement}

For the EV failing the requirements of SAE J551-5, a numerical model has been set up to predict its radiated low-frequency EMI and validated by comparison with the 


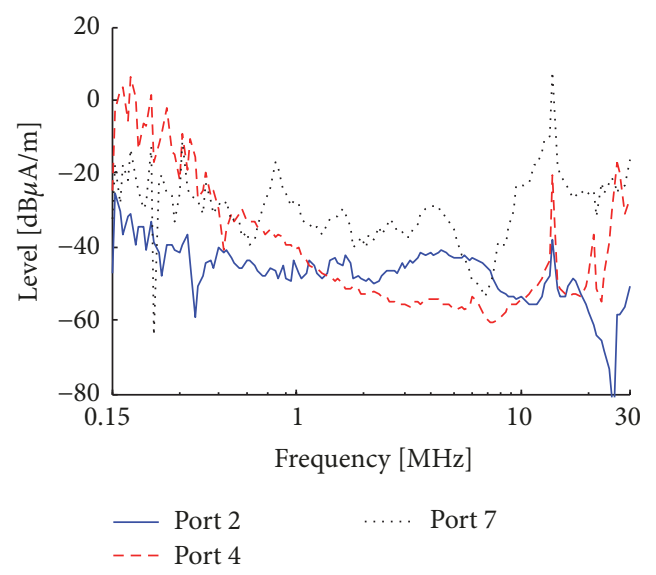

FIGURE 9: EMI from the three maximum components.

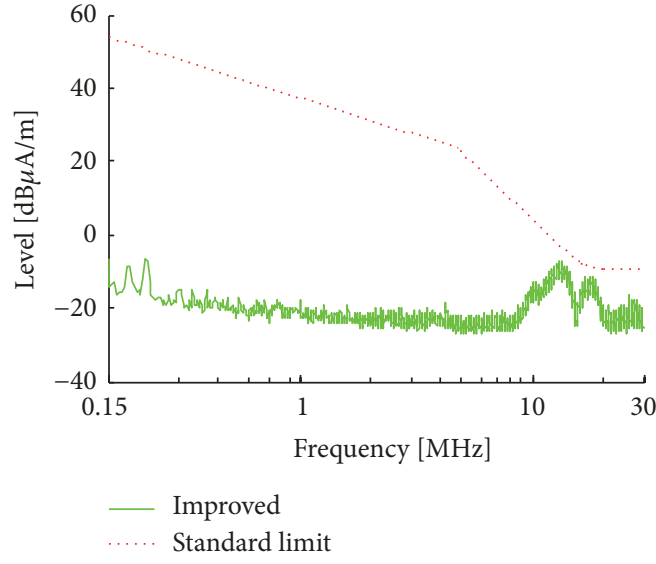

(a)

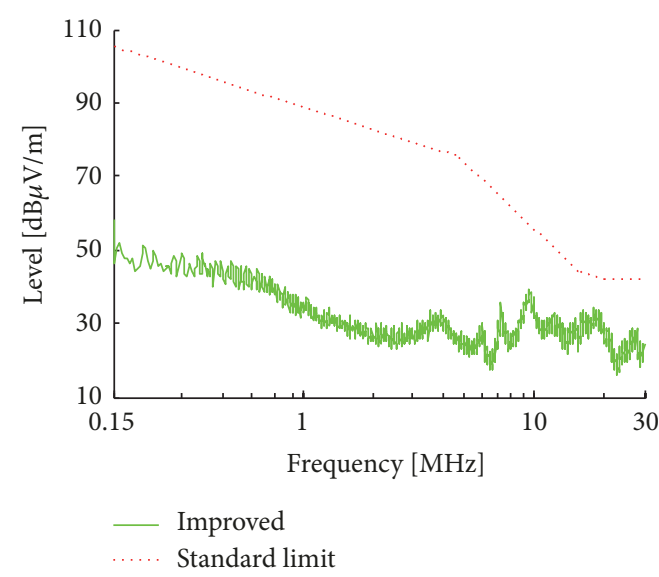

(b)

FIGURE 10: Test result after improvement: (a) magnetic field; (b) electric field.

measurement results. Instead of identifying the main source of EMI by expensive experimental testing, this predictive model is used to analyze the contribution to vehicle-level radiated EMI from each electronic component. Equation (5) shows that the vehicle-level radiated EMI is a sum of the induced interferences from every source. Then the generated interference magnetic field of each component can be derived, which is shown in Figure 9. Note that only the three maximum EMI sources are plotted to show clearly.

It is found that at the lower frequency range the greatest EMI is the drive inverter, whereas the EMI generated by the DC/DC is much higher than others especially at around the frequency where the EMI exceeds the limit. Therefore, this predictive model indicates that the DC/DC is the main source leading to excess vehicle-level magnetic field emission.

Although the radiated emission are also influenced by the coupling paths, from the standpoint of automotive enterprises, the easiest way is to improve EMC of the DC/DC converter because the vehicle may be required to be rearranged to reduce the coupling between components. This is because $\mathbf{S}$-parameter and $\mathbf{Z}$-parameter are affected by many factors like the structure of vehicle body, the layout of cables, and so on, and it is difficult to control the variation of them. Note that improvement of EMC is at the last stage of vehicle development process, so we should consider the easiest method first. If it failed, redesigning the electric system or vehicle body is the last resort.

After redesigning the DC/DC, the new test results of vehicle-level low-frequency emission according to SAE J551-5 are shown in Figure 10, which show that the EV can pass SAE J551-5. This implies that the troubleshooting result obtained by the presented numerical method is correct and effective. Compared with the original experimental method, the cost of both time and money is greatly reduced.

\section{Conclusion}

This paper proposes a methodology for improving vehiclelevel radiated emission by numerical analysis. This methodology combines both simulation in electromagnetic numerical solving software and measurement in actual vehicle to predict the vehicle-level radiated emission. Then this model is applied to find out the main EMI source. The experimental application results show that the radiated emission of the EV is improved successfully with reduced cost. 
This methodology can be used not only in the improvement of vehicle-level radiated EMI, but also in other kinds of EMC issues, which can be equivalent to a multiport network. However, there are some open problems that need to be further studied:

(1) The characteristics of electronic/electrical components may vary with working conditions. Theoretically, the parameters of the equivalent circuit should be obtained under the condition, which is the same with the studied EMC problem.

(2) The transmission path of the presented application is approximated to be linear. How to model and solve the nonlinear interactions is to be studied.

\section{Data Availability}

We can provide the following data in the article: (1) the experimental test results of the original studied vehicle according to J551-5; (2) the data used to predict the low-frequency EMI including tested impedance data of components, the more common interference current of the EMI source port, the Sparameters of the equivalent network calculated by FEKO, and the factors of measuring antennas; (3) the experimental test results of the improved vehicle whose DC/DC is modified. The data are available from the corresponding author upon request. Unfortunately, we cannot provide the original FEKO project used to calculate the S-parameters, because it includes the vehicle model belonging to some OEM.

\section{Conflicts of Interest}

The authors declare that they have no conflicts of interest.

\section{Acknowledgments}

This research was supported the National Key R\&D Program of China under grant 2017YFB0102504 and Scientific Technological Plans of Chongqing under grant cstc2017zdcyzdzx0042. Thanks are due to the China Automotive Technology and Research Center, who provided the EV and the EMC laboratory.

\section{References}

[1] D. Hamza and M. Pahlevaninezhad, "Integrated capacitor for common-mode EMI mitigation applicable to high frequency planar transformers used in electric vehicles DC/DC converters," in Proceedings of the 2014 IEEE Energy Conversion Congress and Exposition (ECCE' 14), pp. 4809-4814, Pittsburgh, Pa, USA, 2014.

[2] S. Jeschke, H. Hirsch, M. Trautmann et al., "EMI measurement on electric vehicle drive inverters using a passive motor impedance network," in Proceedings of the 7th Asia-Pacific International Symposium on Electromagnetic Compatibility, APEMC '16, pp. 292-294, Shenzhen, China, 2016.

[3] H. Shim, H. Kim, Y. Kwack et al., "Inverter modeling including non-ideal IGBT characteristics in Hybrid Electric Vehicle for accurate EMI noise prediction," in Proceedings of the 2015 IEEE International Symposium on Electromagnetic Compatibility EMC '15, pp. 691-695, Dresden, Germany, 2015.
[4] A. R. Ruddle and R. Armstrong, "Review of current EMC standards in relation to vehicles with electric powertrains," in Proceedings of the 2013 International Symposium on Electromagnetic Compatibility, EMC Europe '13, pp. 298-303, Brugge, Belgium, September 2013.

[5] M. C. Di Piazza, M. Luna, A. Ragusa, and G. Vitale, "An improved common mode active filter for EMI reduction in vehicular motor drives," in Proceedings of the 7th IEEE Vehicle Power and Propulsion Conference, VPPC '11, pp. 1-8, Chicago, Ill, USA, 2011.

[6] C. Marczok, U. Maaß, E. Hoene, I. Ndip, K.-D. Lang, and D. Hasselberg, "Analysis and improvement of a spark plug for less radiated electromagnetic emissions," in Proceedings of the 2014 International Symposium on Electromagnetic Compatibility, EMC Europe '14, pp. 385-390, Gothenburg, Sweden, 2014.

[7] H. Chun et al., "Converter Switching Noise Reduction for Enhancing EMC Performance in HEV and EV," in Proceedings of the PCIM Europe 2016; International Exhibition and Conference for Power Electronics, Intelligent Motion, Renewable Energy and Energy Management, pp. 1-8, Nuremberg, Germany, 2016.

[8] G. Ala, M. C. Di Piazza, G. Tinè, F. Viola, and G. Vitale, "Evaluation of radiated EMI in $42-\mathrm{V}$ vehicle electrical systems by FDTD simulation," IEEE Transactions on Vehicular Technology, vol. 56, no. 4 I, pp. 1477-1484, 2007.

[9] C. Chen, "Predicting vehicle-level radiated EMI emissions using module-level conducted EMIs and harness radiation efficiencies," in Proceedings of the 2001 International Symposium on Electromagnetic Compatibility (EMC '01), pp. 1146-1151, Montreal, Canada, 2001.

[10] H. Zeng, I. Hoda, W. Ivan et al., "Vehicle-Level EMC Modeling for HEV/EV Applications," SAE International Journal of Passenger Cars - Electronic and Electrical Systems, vol. 8, no. 2, pp. 278281, 2015.

[11] M. Dong, L. Zhai, R. Gao, and X. Zhang, "Research on radiated electromagnetic interference (EMI) from power cables of a three-phase inverter for electric vehicles," in Proceedings of the 2014 IEEE Transportation Electrification Conference and Expo Transportation Electrification Asia-Pacific, pp. 1-5, Beijing, China, 2014. 


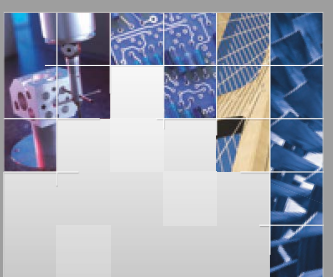

\section{Enfincering}
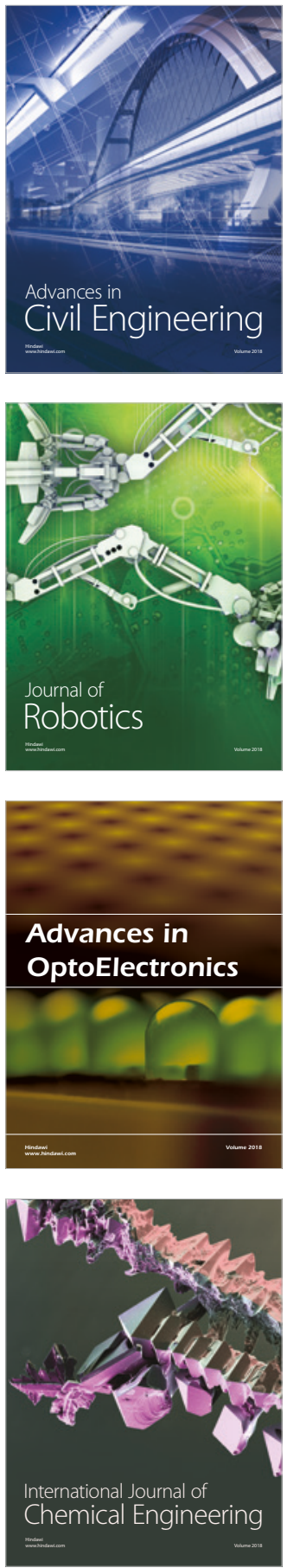

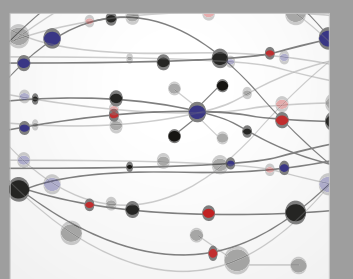

\section{Rotating \\ Machinery}

The Scientific World Journal

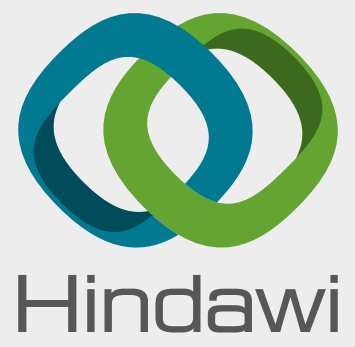

Submit your manuscripts at

www.hindawi.com
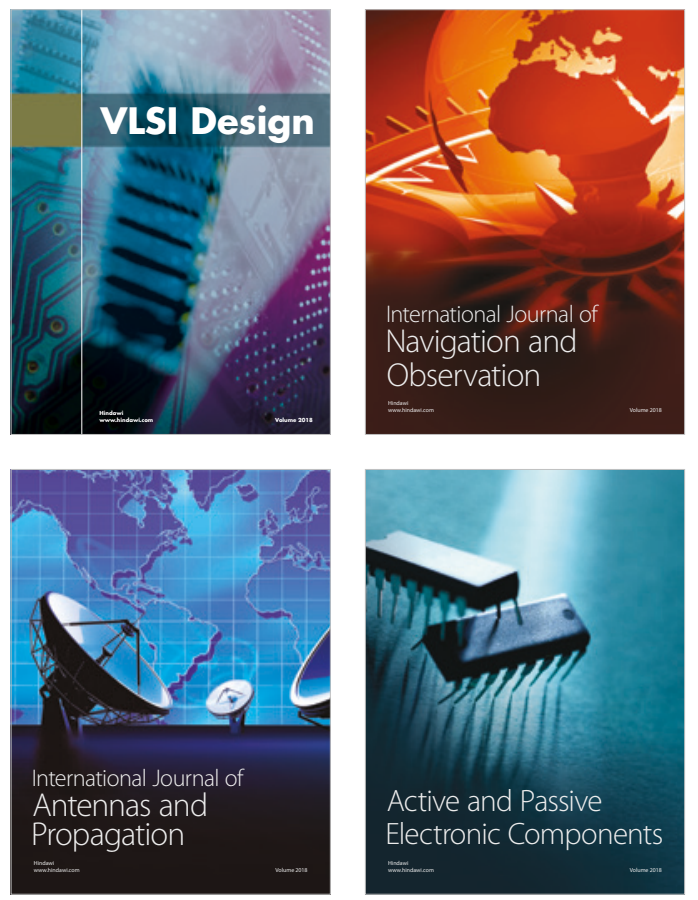
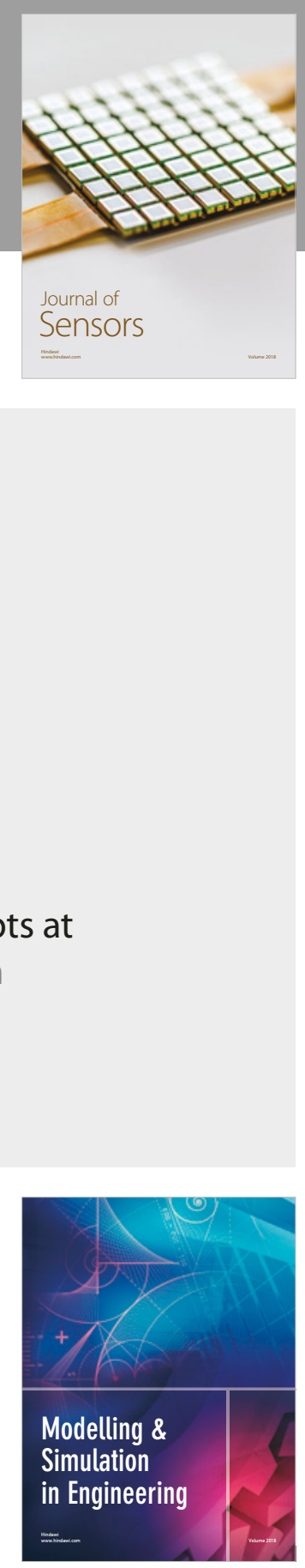

\section{Advances \\ Multimedia}
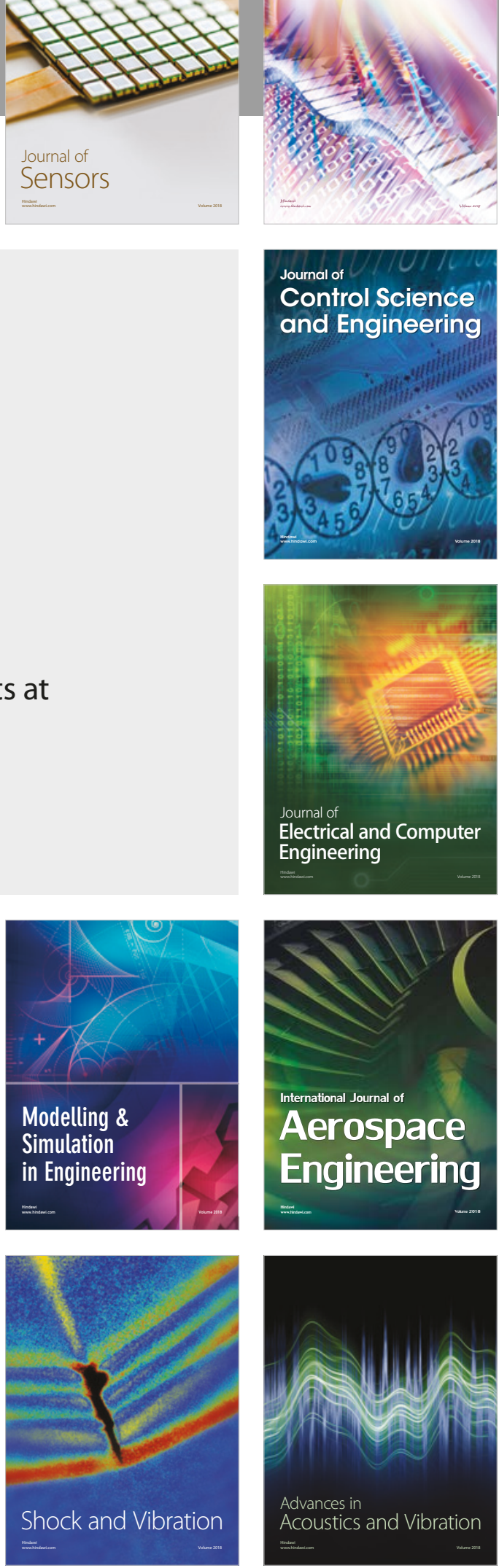